\title{
Non-Stationary Spectral Estimation for Wind Turbine Induction Generator Faults Detection
}

\author{
El Houssin El Bouchikhi ${ }^{1}$, Vincent Choqueuse ${ }^{1}$ and M.E.H. Benbouzid ${ }^{1}$ \\ ${ }^{1}$ University of Brest, EA 4325 LBMS, Rue de Kergoat, CS 93837, 29238 Brest Cedex 03, France
}

\begin{abstract}
Development of large scale offshore wind and marine current turbine farms implies to minimize and predict maintenance operations. In direct- or indirect-drive, fixed- or variable-speed turbine generators, advanced signal processing tools are required to detect and diagnose the generator faults from vibration, acoustic, or generator current signals. The induction generator is traditionally used for wind turbines power generation. Even if induction machines are highly reliable, they are subjected to many types of faults. The aim then, is to detect them at an early stage in order to prevent breakdowns and consequently ensure the continuity of power production. In this context, this paper deals with wind turbines condition monitoring using a fault detection technique based on the generator stator current. The detection algorithm uses a recursive maximum likelihood estimator to track the time-varying fault characteristic frequency and the related energy. Furthermore, a decisionmaking scheme and a related criterion are proposed.

The feasibility of the proposed approach has been demonstrated using simulation data issued from coupled magnetic circuits induction generator model driven by a wind turbine for both electrical asymmetry and mechanical imbalance.
\end{abstract}

Index Terms-Wind turbines, induction machine, faults detection, stator current, spectral estimation, maximum likelihood estimator.

\section{INTRODUCTION}

Diagnosis and condition monitoring are effective means to improve wind turbine reliability and performance and reduce wind turbine operating and maintenance costs. They are one of the huge issues that face the deployment of offshore systems such as wind or marine current turbines [1]-[3]. In fact, the availability and the reliability of these systems decrease significantly when they are settled in the ocean [4].

Most existing technologies for wind turbine condition monitoring require additional sensors and data acquisition devices to be implemented [5]. The use of these sensors and devices increases cost, size, and hardware wiring complexity of the wind turbine condition monitoring system. Most of these sensors are mounted on the surface or are buried in the body of wind turbine components, which are difficult to access during wind turbine operation. Therefore, it is desirable to develop a non-intrusive, lower-cost, and more reliable technology to fully exploit the benefits of condition monitoring for wind turbines.

In the last few years, many studies demonstrated the interest of using the stator current to monitor induction generators and therefore the associated wind turbine [6], [7]. In previous works, wind turbine faults detection was performed using advanced signal processing techniques such as demodulation techniques [8], [9] and time-frequency techniques [10], [11]. Moreover, adaptive fault frequency tracking techniques have been proposed for stator current based fault detection in wind generators [12], [13]. However, these methods were adopted without concerns about the signal nature (multi-component signals, non-stationary signals, etc.) nor about their performances. Many techniques have been proposed and, in some practical situations, it is not easy to select the most appropriate one. Moreover, These papers do not present a post-processing algorithms allowing to extract a fault indicator from these stator current time-frequency techniques. If good performances are required, the easiness of results interpretation is also a key feature for a future implementation in a real-time monitoring system.

This paper presents a current-based technique for online wind turbine condition monitoring. The proposed technique uses only phase current signals measured from generator stator terminals for the wind turbine condition monitoring. Since these current measurements are used by existing wind turbine control systems, no additional sensors or data acquisition devices are required to implement the proposed technique. This technique is able to effectively extract the wind turbine fault or failure from current measurements using adaptive fault related frequency estimation. Afterwards, recursive algorithm is used in order to extract a fault severity indicator. Simulation results prove that the proposed technique offers an effective mean to achieve condition-based, nonintrusive, and smart maintenance for wind turbines.

\section{ADAPTIVE TIME-FREQUENCY TECHNIQUE AS MEDIUM FOR FAULT DETECTION IN WIND GENERATOR}

\section{A. Wind Generator Stator Current Model}

The induction machine stator current $x[n]$ in presence of mechanical and/or electrical faults can be expressed as follows

$$
x[n]=\sum_{k=-L}^{L} a_{k} \cos \left(2 \pi f_{k}(\Omega(n)) \times\left(\frac{n}{F_{s}}\right)+\phi_{k}\right)+b[n]
$$

where $x[n]$ corresponds to the $n^{\text {th }}$ stator current sample, $b[n]$ corresponds to the noise and $L$ is the number of sidebands introduced by the fault. The parameters $f_{k}(\Omega), a_{k}$ and $\phi_{k}$ correspond to the frequency, the amplitude and the phase of the $k^{t h}$ component, respectively. Symbol $F_{S}$ corresponds to 
the sampling frequency and $\Omega(n)$ is a set of parameters to be estimated at each time $n$ depending on the fault studied. It worth to notice that the time and space harmonics are not considered but it is possible to met the signal model in (1) by filtering the original signal using a low-pass anti-aliasing filter.

At time $\mathrm{n}=0,1,2,3 \ldots$ the observed stator current vector $\mathbf{x}(n) \in$ $\mathbb{R}^{m}$, defined as $\mathbf{x}(n)=[x(n) \ldots x(n+M-1)]^{T}$, can be expressed as

$$
\mathbf{x}(n)=\mathbf{A}(\Omega(n)) \mathbf{v}(n)+\mathbf{b}(n)
$$

where:

$-\mathbf{x}(n)=[x[n], \cdots, x[n+M-1]]^{T}$ is a $M \times 1$ column vector containing the stator current samples,

- $\mathbf{b}(n)=[b[n], \cdots, b[n+M-1]]^{T}$ is a $M \times 1$ column vector containing the noise samples,

$-\mathbf{v}(n)$ is a $2(2 L+1) \times 1$ column vector containing the amplitudes and phases of the characteristic fault frequencies. This vector is given by

$$
\begin{aligned}
\mathbf{v}(n)=\left[a_{-L}\right. & \cos \left(\phi_{-L}\right) \ldots a_{L} \cos \left(\phi_{L}\right), \\
& \left.-a_{-L} \sin \left(\phi_{-L}\right) \ldots-a_{L} \sin \left(\phi_{L}\right)\right]^{T}
\end{aligned}
$$

- $\mathbf{A}(\Omega(n))$ is a $N \times 2(2 L+1)$ matrix given by

$$
\mathbf{A}(\Omega(n))=\left[\mathbf{z}_{-L}(n) \ldots \mathbf{z}_{L}(n), \mathbf{y}_{-L}(n) \ldots \mathbf{y}_{L}(n)\right]
$$

where:

$$
\begin{aligned}
& \mathbf{z}_{k}(n)= {\left[\begin{array}{c}
1 \\
\cos \left(2 \pi f_{k}(\Omega(n)) \times \frac{1}{F_{s}}\right) \\
\vdots \\
\cos \left(2 \pi f_{k}(\Omega(n)) \times \frac{M-1}{F_{s}}\right)
\end{array}\right] } \\
& \mathbf{y}_{k}(n)=\left[\begin{array}{c}
\sin \left(2 \pi f_{k}(\Omega(n)) \times \frac{1}{F_{s}}\right) \\
\vdots \\
\sin \left(2 \pi f_{k}(\Omega(n)) \times \frac{M-1}{F_{s}}\right)
\end{array}\right]
\end{aligned}
$$

- $\Omega(n)$ is a set of parameters to be estimated depending on the fault.

- The symbol (.) $)^{T}$ corresponds to the matrix transpose.

The problem is then to estimate the fundamental frequency, the fault characteristic frequency, and their amplitudes for fault characterization. The computation of the current spectrum shown in Fig. 1 from stator current samples $\mathbf{x}(n)$ is treated as a statistical estimation problem. The observation noise $\mathbf{b}(n)$ is assumed to be zero-mean white Gaussian distributed with variance $\sigma^{2}$.

\section{B. Fault Characteristic Frequency and Fault Detection Crite- rion Estimation}

In this section, we present a maximum likelihood (ML) based fault characteristic frequency estimation. We consider

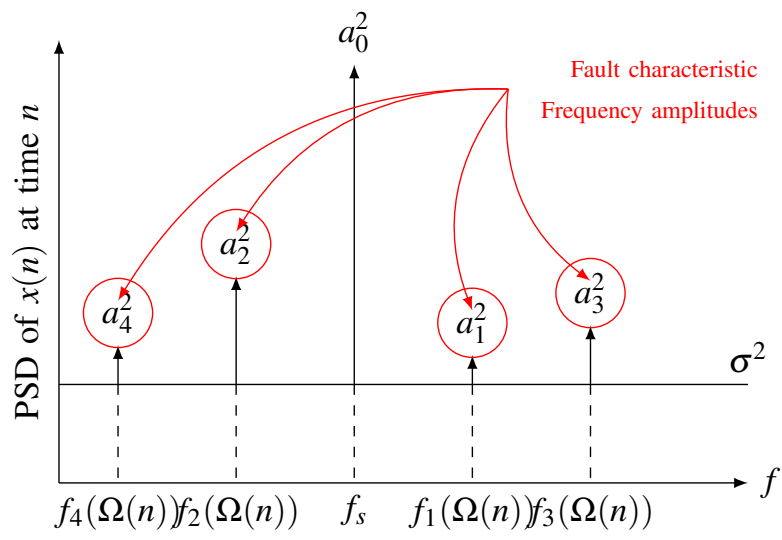

Fig. 1. Theoretical PSD for $L=2$ [14].

how to solve the optimization problem in a computationally simple manner. Then, we explore a fault criterion estimation for decision making.

1) Estimate of $\Omega(n)$ : The algorithm operates on a signal sub-vectors at times n. Although the approach of splitting the signal into sub-vectors is inherently suboptimal since it ignores inter-vector dependencies, it is required in order to estimate signal and noise covariance matrices. For multiple observation vectors, the ML estimate of $\Omega(n)$ can be shown to be the maximizer of the cost function [15]

$$
\mathscr{J}(\Omega(n))=-\operatorname{Tr}\left\{\mathbf{A}(\Omega(n)) \mathbf{A}^{\dagger}(\Omega(n)) \mathbf{R}(n)\right\}
$$

with $\mathbf{A}^{\dagger}(\Omega(n))$ is the pseudo-inverse of $\mathbf{A}(\Omega(n))$ i.e.

$$
\mathbf{A}^{\dagger}(\Omega(n))=\left(\mathbf{A}^{T}(\Omega(n)) \mathbf{A}(\Omega(n))\right)^{-1} \mathbf{A}^{T}(\Omega(n))
$$

where, (. $)^{-1}$ corresponds to the matrix inverse, and $\operatorname{Tr}($. denotes the matrix trace.

The covariance matrix $\mathbf{R}(\mathrm{n})$ of the observed signal is given by

$$
\mathbf{R}(n)=E\left\{\mathbf{x}(n) \mathbf{x}^{T}(n)\right\}
$$

The proposed methodology relies on this covariance matrix. In practice, the covariance matrix is unknown and must be replaced by its estimate, the sample covariance matrix, i.e.,

$$
\widehat{\mathbf{R}}(n)=\frac{1}{G} \sum_{n=0}^{G-1} \mathbf{x}(n) \mathbf{x}^{T}(n)
$$

Since $\mathbf{x}[n]$ has length $M$ and we have $N$ observations of $x[n]$, we can thus construct $G=N-M+1$ different subvectors $\{\mathbf{x}[n]\}_{n=0}^{G-1}$. There is some inherent tradeoffs in choices of $N$ and $M$ and thereby the number of the sub-vectors $G$ [15].

Finally, $\Omega(n)$ can be estimated from this cost function as

$$
\{\widehat{\Omega}(n)\}=\arg \max _{\Omega} \mathscr{J}(\Omega(n))
$$

This estimator is capable of handling non-stationary signals since the covariance matrix is estimated in recursive manner by dividing the signal into time segments and then, the covariance 
matrix is estimated with $50 \%$ overlap between stator current segments $\mathbf{x}[n]$.

2) Estimation of $L$ : The fault diagnosis comprise two steps; the fault detection and the fault isolation. The estimation of $L$ allows to distinguish the faulty machine from the healthy one. In this paper, we propose an approach based on the Minimum Description Length (MDL) principle [16] for $L$ estimation in order to enhance fault detection and afterwards accuracy while estimating the fault severity.

$$
\begin{aligned}
\{\widehat{\Omega}(n), \widehat{L}(n)\}=\arg \max _{\Omega, L} & -\operatorname{Tr}\left\{\left(I_{M}-\mathbf{A}(\Omega(n)) \mathbf{A}^{\dagger}(\Omega(n))\right)\right. \\
& \mathbf{R}(n)\} \times \exp \left(\frac{c(g, M)}{M}\right)
\end{aligned}
$$

with $c(g, M)=g \log (M)$ is the criterion information rule, and $g=4 L+5$ is the number of free parameters. Furthermore, $I_{M}$ is the identity matrix of size $M$.

3) Fault detection criterion: The ML estimate of $\mathbf{v}(n)$ denoted $\widehat{\mathbf{v}}(n)$ is given by [15]

$$
\widehat{\mathbf{v}}=\mathbf{A}^{\dagger}(\widehat{\Omega}(n)) \mathbf{x}
$$

Since the information about the fault severity is carried out by $a_{k}(k \neq 0)$, we propose to compute the sum of the squares of the (normalized) amplitude of the fault characteristic frequencies. This criterion is mathematically expressed as

$$
\mathscr{C}(n)=\sum_{k=-L, k \neq 0}^{L}\left(\frac{a_{k}^{2}}{a_{0}^{2}}\right)
$$

Using the structure of $\mathbf{v}(n)$ in (3), it can be shown that $\mathscr{C}(n)$ can be obtained directly from $\mathbf{v}(n)$ without computing $a_{k}$. Indeed, the fault criterion in (12) can be expressed under the following matrix form:

$$
\mathscr{C}(n)=\frac{\mathbf{v}^{T}(n) \mathbf{v}(n)}{\mathbf{v}^{T}(n) \mathbf{M} \mathbf{v}(n)}-1
$$

where $\mathbf{M}$ is a $(4 L+2) \times(4 L+2)$ matrix which is given by

$$
\left[\begin{array}{cc}
\mathbf{E}_{L+1, L+1} & \mathbf{0} \\
\mathbf{0} & \mathbf{E}_{L+1, L+1}
\end{array}\right]
$$

and where $\mathbf{E}_{u, v}$ is the $(2 L+1) \times(2 L+1)$ elementary matrix which is 1 in the $u^{\text {th }}$ row and $v^{\text {th }}$ column and is zero elsewhere. In practice, one should note that $\mathbf{v}(n)$ is unknown and must be replaced by its estimate $\widehat{\mathbf{v}}(n)$ in (13) to compute $\mathscr{C}(n)$.

The fault detection approach is summed up in Algorithm 1.

\section{Numerical Example}

Synthetic signals $\mathbf{x}(n)$ were simulated by using the signal model described in Fig. 1. We consider the case where the fault characteristic frequency is given by $f_{k}=f_{s}+k f_{c}(k \in \mathbb{Z})$ and where $f_{s}=50 \mathrm{~Hz}, f_{c}=10 \mathrm{~Hz}, L=1$, and $S N R=50 \mathrm{~dB}$. The set $\Omega=\left\{f_{s}(n), f_{c}(n), L(n)\right\}$ constitutes the parameters to be estimated. The cost function in (5) is shown in Fig. 2 for a time segment of a signal $\mathbf{x}(n)$ described by the model given $\overline{\text { Algorithm } 1 \text { Non stationary estimation-based failure severity }}$ criteria.

\section{Require: $\mathbf{N}$-stator current data samples $\mathbf{x}[n]$.}

1: Estimate the sample covariance matrix using (8).

2: Compute the MLE exact cost function in (10),

3: Optimization procedure in order to find $\widehat{\Omega}(n)$ and $\widehat{L}(n)$,

$$
\begin{aligned}
& \text { if } \widehat{L}(n)=0 \text { then } \\
& \text { fault }=0 \\
& \mathscr{C}(n)=0 \\
& \text { else }
\end{aligned}
$$

fault $=1$

Estimate $\widehat{\mathbf{v}}(n)$ with (11)

Compute fault detection criterion $\mathscr{C}(n)$ using (13) end if

4: Return $\widehat{\Omega}, \widehat{L}$, fault, $\widehat{\mathbf{v}}$, and $\mathscr{C}$.

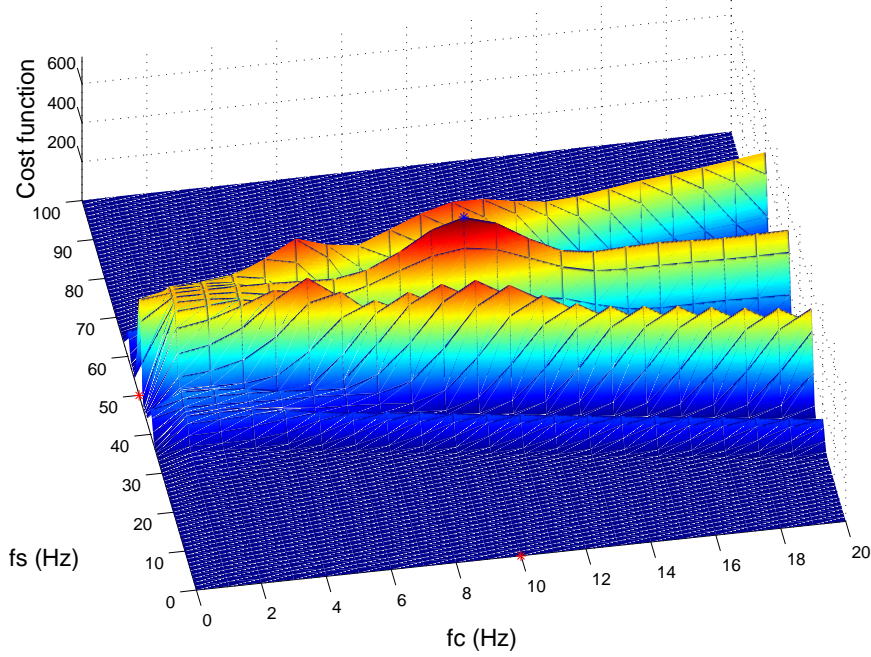

Fig. 2. Cost function at time $n$ for $f_{c}$ and $f_{s}$ estimation (case where fault exists and $L(n)=1$ )

by (1). It can be seen that it allows to determine the supply frequency as well as the fault characteristic frequency.

For incipient fault detection performance, we propose to apply the proposed approach on signal with no fault until $2 s$, then a fault occur with time-varying related fault frequency ( $f_{c}=10 \mathrm{~Hz}$ until $4 \mathrm{~s} \mathrm{~Hz}$, then $f_{c}=15 \mathrm{~Hz}$ ), sidebands number and severity increasing. The simulation results are shown in Fig. 4 for the estimation of $L, f_{c}$, and $f_{s}$. Figure 3 displays the time-frequency representation using the spectrogram and the proposed methodology as well as the proposed fault detection criterion.

It can be concluded that the proposed method allows tracking the fault related frequency and the number of sidebands. In contrary to the spectrogram, the proposed approach is more accurate since it allows to detect 4 sidebands around the supply frequency for $4<t<6$. This adaptive fault detection method will be used in the following for wind turbine induction generator fault detection using simulated stator current. 


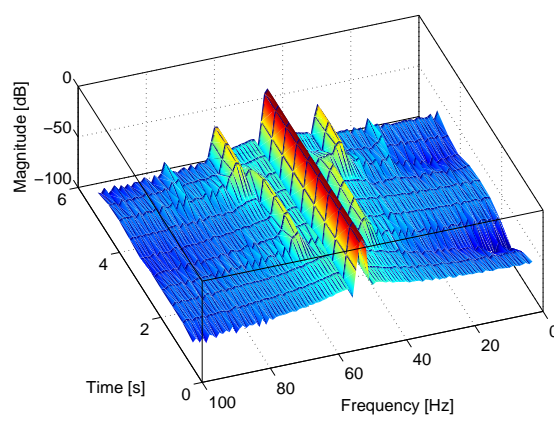

(a) Spectrogram.

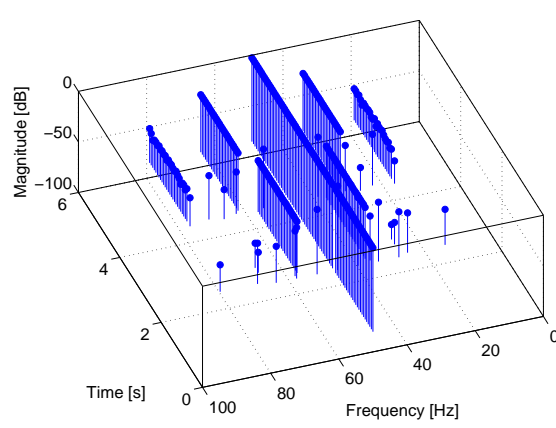

(b) Proposed time-frequency representation.

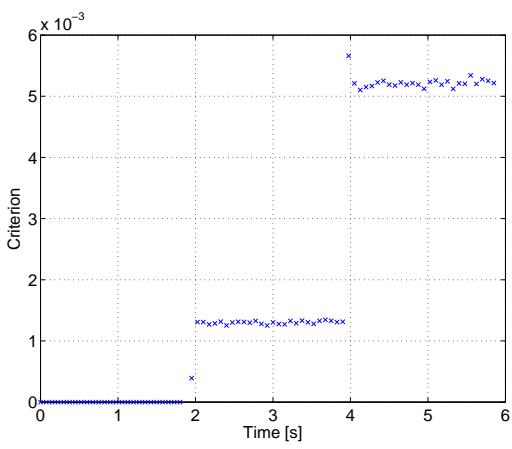

(c) Proposed fault detection criterion

Fig. 3. Time-frequency representation and proposed fault criterion variation.
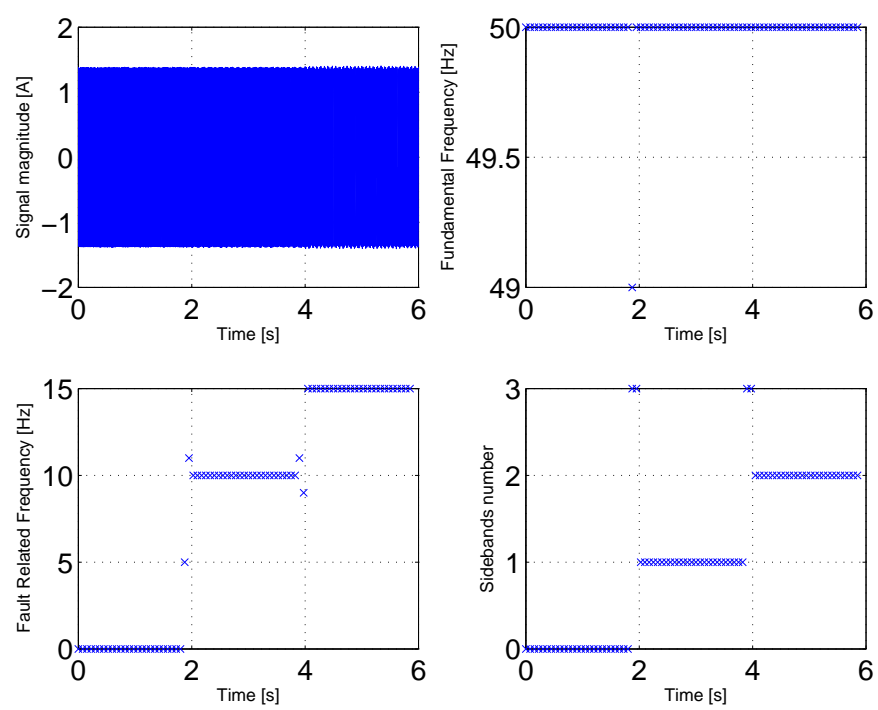

Fig. 4. Fundamental frequency, fault related frequency and sidebands number estimation.

\section{APPLICATION TO WIND TURBINE FAULTS DETECTION}

The proposed approach performance is evaluated using simulated stator currents issued from a Matlab-Simulink ${ }^{\circledR}$. based simulation tool of healthy and faulty induction machine [17]. In this context, a $4 \mathrm{~kW}$ induction generator is used and is driven by a wind turbine. The proposed technique is evaluated for rotor imbalance and broken rotor bar faults detection.

\section{A. Induction Generator Modelling Briefly}

For most faults, the harmonic contents of the stator current can be calculated satisfactory using linear models of the machine such as the coupled magnetic circuit method. This approach is based on the analytical equations of the induction machine. All parameters are calculated from the actual geometry and winding layout of the machines rather than from transformed or equivalent variables. This analysis is based on some assumptions: infinite iron permeability, non conductive magnetic circuit, no inter-bars currents and negligible saturation. The detailed description of this model can be found in [17]-[19].

The simulation has been performed using 2 pair poles, a $4 \mathrm{~kW} / 50 \mathrm{~Hz}, 230 / 400$.induction machine. The induction machine model was operated as generator driven by a wind turbine. The model was operated for $10 s$ and the fault appears at $5 s$. The simulation results; the mechanical speed, the electromagnetic torque, and the stator current are given by Fig. 5 for one broken rotor bar.

In this paper, we investigate the performance of the proposed fault detection strategy for mechanical imbalance fault as it concerns approximately $80 \%$ of the mechanical faults [20], [21] and the broken rotor bars as it is one of the electrical asymmetries that is difficult to detect since the squirrel cage currents are not directly accessible.

\section{B. Simulation Results}

1) Electrical asymmetry detection: Computer simulations have been performed to assess the operating features of the proposed fault detection scheme for $0,1,2$, and 3 broken rotor bars. The simulations have been done for adjacent and non-adjacent broken rotor bars.

It can be seen from Fig. 6 that the proposed methodology provides a reliable way to track the fault related frequency based on the stator current. Moreover, it allows to track the fault severity which gives to the operator an idea about the health state of the induction generator and the associated turbine. This shows the potential of the proposed approach to detect incipient electrical asymmetry faults on a wind turbine generator.

2) Mechanical imbalance fault detection: The mechanical imbalance fault was simulated by considering static, dynamic, and mixed eccentricity. In fact, the non-uniformity between the stator and the rotor leads to air gap eccentricity. In this simulations, $5 \%$ eccentricity fault is considered until $5 s$, then the severity increases to $10 \%$ air gap eccentricity. Figures 7 , 8 and 9, give the simulation results for static, dynamic and mixed eccentricities detection using the proposed algorithm.

These results demonstrate the effectiveness of the proposed approach for electrical fault detection in induction machine operated as generator. Even if wind turbulence influence 


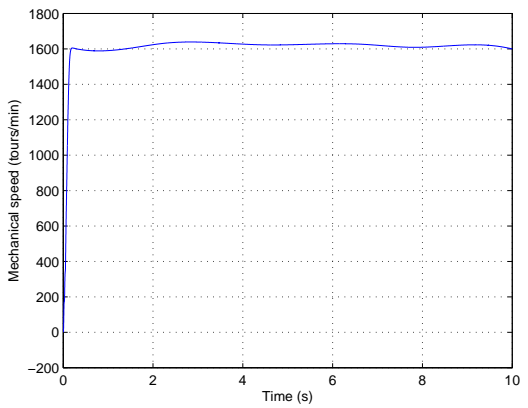

(a) Induction generator mechanical speed.

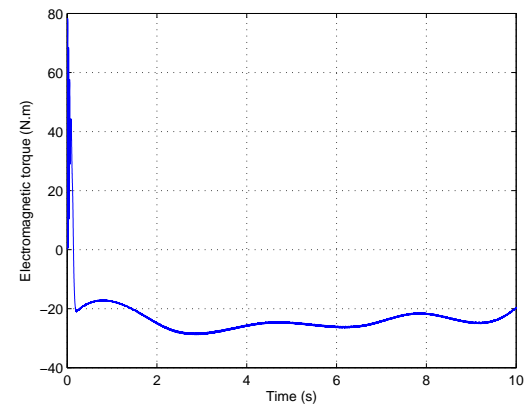

(b) Induction generator electromagnetic torque

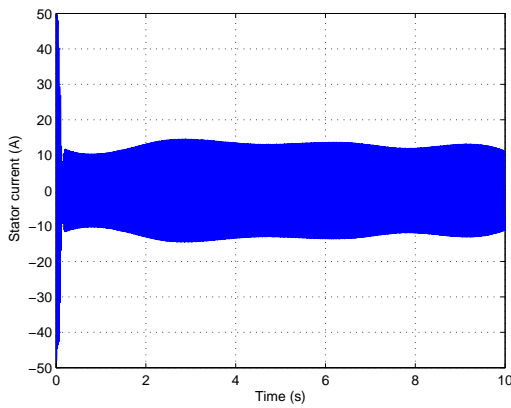

(c) Induction generator stator current.

Fig. 5. Faulty induction generator-based wind turbine simulation signals.

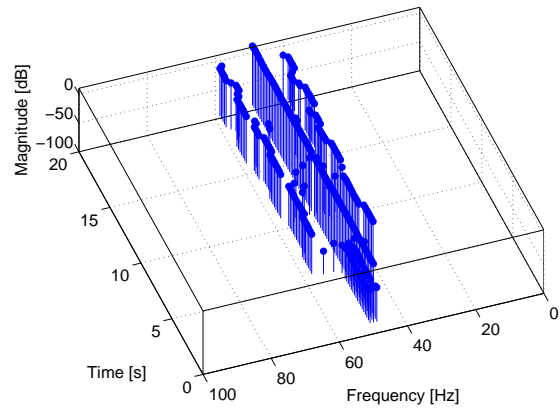

(a) Time-frequency representation.

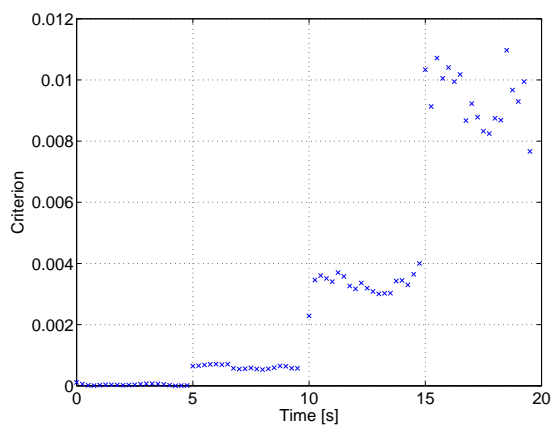

(b) Fault detection criterion tracking.

Fig. 6. Fault severity tracking using the proposed approach; 1, 2, and 3 bars has been broken consecutively.

(amplitude modulation seen in the time domain) tends to hide the fault influence on the stator current especially for incipient fault, the proposed approach demonstrated good performance and allows to measure the fault severity. As compared to other time-frequency techniques [10], the proposed approach is developed to meet the requirement of fault detection which are the fault characteristic frequency tracking and fault severity measurement.

\section{CONCLUSION}

To improve wind turbines (WT) availability and reduce operating and maintenance costs, this paper has proposed a new WT condition monitoring technique. From the achieved simulation results, it can be concluded that the proposed

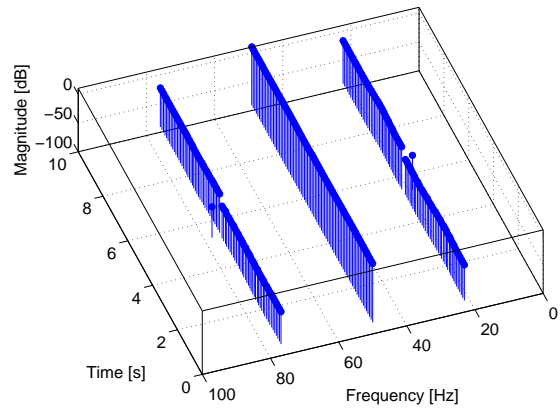

(a) Time-frequency representation.

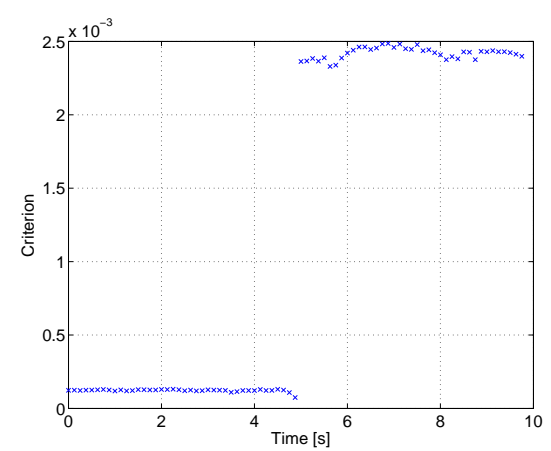

(b) Fault detection criterion tracking.

Fig. 7. Fault severity tracking using the proposed approach; static eccentricity.

method is able to detect both electrical and mechanical faults using stator current processing. In fact, the maximum likelihood-based fault related frequency tracking provides a feasible condition monitoring approach applicable to WTs generators operating at either fixed or variable speed.

Further investigations should be performed in order to validate the approach proposed on power signals issued from induction generators and from real WTs operating under mechanical and electrical faults. Furthermore, it is mandatory to establish the feasibility of the approach on variable-speed WT (based on DFIG and PMSG) under closed-loop control. 


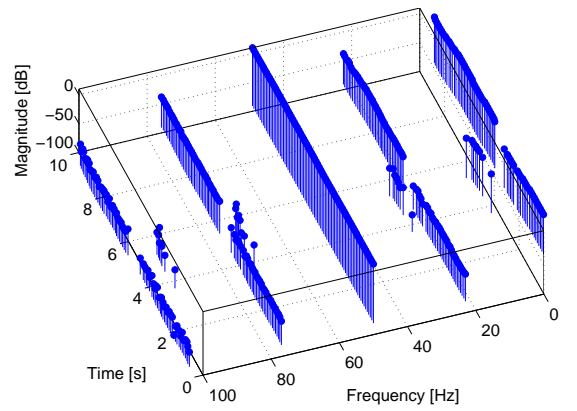

(a) Time-frequency representation.

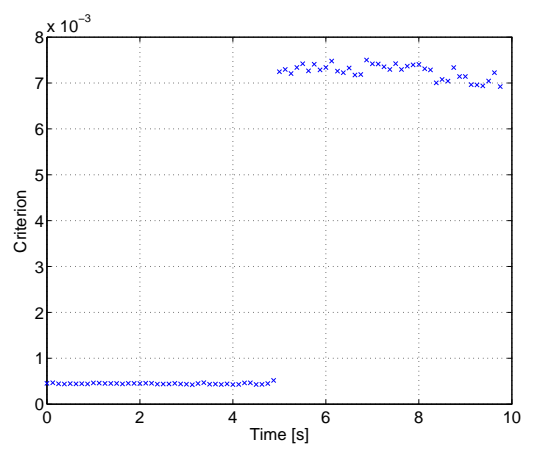

(b) Fault detection criterion tracking.

Fig. 8. Fault severity tracking using the proposed approach; dynamic eccentricity.

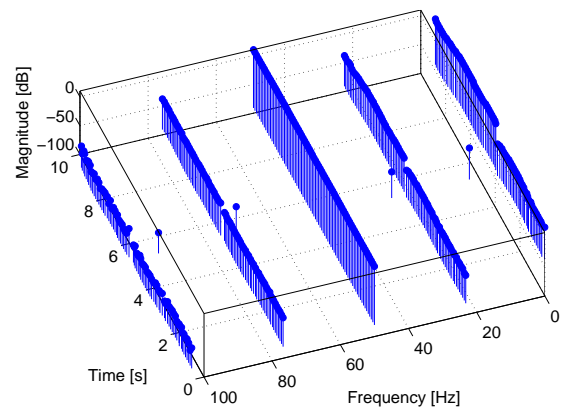

(a) Time-frequency representation.

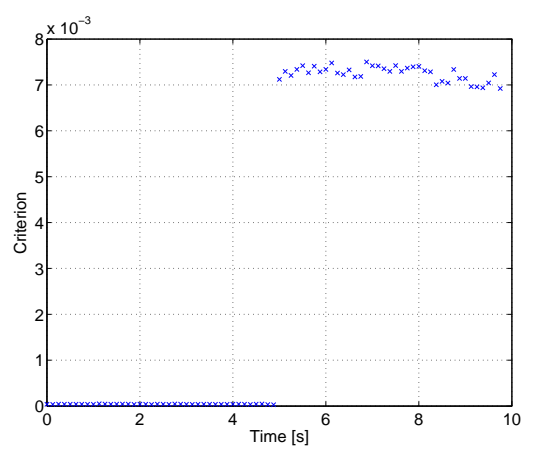

(b) Fault detection criterion tracking.

Fig. 9. Fault severity tracking using the proposed approach; mixed eccentricity.

\section{ACKNOWLEDGMENT}

The financial support provided by Brest Métropole Océane (BMO) is gratefully acknowledged.

\section{REFERENCES}

[1] W. Yang, P. Tavner, C. Crabtree, and M. Wilkinson, "Cost-effective condition monitoring for wind turbines," Transactions on Industrial Electronics, vol. 57, no. 1, pp. 263-271, January 2010.

[2] S. Faulstich, B. Hahn, and P. Tavner, Wind turbine downtime and its importance for offshore deployment. Wing Energy, DOI: 10.1002/we.421, July 2010.

[3] A. Kusiak and W. Li, "The prediction and diagnosis of wind turbine faults," Renewable Energy, vol. 36, no. 1, pp. 16-23, January 2010.

[4] J. J. Nielsen and J. D. Srensen, "On risk-based operation and maintenance of offshore wind turbine components," Reliability Engineering \& System Safety, vol. 96, no. 1, pp. 218-229, January 2011.

[5] Z. Hameed, Y. Hong, Y. Cho, S. Ahn, and C. Song, "Condition monitoring and fault detection of wind turbines and related algorithms: A review," Renewable and Sustainable Energy Reviews, vol. 13, no. 1, pp. 21-39, January 2009.

[6] X. Gong and W. Qiao, "Imbalance fault detection of direct-drive wind turbines using generator current signals," IEEE Trans. Energy Conversion, vol. 27, no. 2, pp. 468-476, June 2012.

[7] M. E. H. Benbouzid, "A review of induction motors signature analysis as a medium for faults detection," IEEE Transactions Industrial Electronics, vol. 47, no. 5, pp. 984-993, October 2000.

[8] N. Q. Hu, L. R. Xia, F. S. Gu, and G. J. Qin, "A novel transform demodulation algorithm for motor incipient fault detection," IEEE Trans. on Instrumentation and Measurement, vol. 60, no. 2, pp. 480-487, February 2011.

[9] Y. Amirat, V. Choqueuse, M. E. H. Benbouzid, and S. Turri, "Hilbert transform-based bearing failure detection in Dfig-based wind turbines," International Review of Electrical Engineering, vol. 6, no. 3, pp. 12491256, June 2011.

[10] E. H. El Bouchikhi, V. Choqueuse, M. E. H. Benbouzid, J. Charpentier, and G. Barakat, "A comparative study of time-frequency representations for fault detection in wind turbine," in Proceedings of the 2011 IEEE IECON, Melbourne (Australia), November 2011, pp. 3584-3589.

[11] S. Rajagopalan, J. A. Restrepo, J. Aller, T. Habetler, and R. Harley, "Nonstationary motor fault detection using recent quadratic timefrequency representations," IEEE Transactions on Industry Applications, vol. 44, no. 3, 2008.

[12] C. J. Crabtree, S. Djurović, P. J. Tavner, and A. C. Smith, "Fault frequency tracking during transient operation of wind turbine generators," in Proceedings of the 2010 IEEE ICEM, Rome, Italy, September 2010, pp. 1-5.

[13] A. Bellini, G. Franceschini, and C. Tassoni, "Monitoring of induction machines by maximum covariance method for frequency tracking," IEEE Transactions on Industry Applications, vol. 42, no. 1, pp. 69-78, January/February 2006.

[14] P. Stoica and R. Moses, Introduction to Spectral Analysis. Prentice Hall, 1997.

[15] M. G. Christensen and A. Jakobsson, Multi-pitch Estimation. vol. 5 of Synthesis Lectures on Speech \& Audio Processing, Morgan \& Claypool Publishers, 2009.

[16] P. Stoica and Y. Seln, "A review of information criterion rules," IEEE Signal Processing Magazine, vol. 21, no. 4, pp. 36-47, July 2004.

[17] E. H. El Bouchikhi, V. Choqueuse, and M. E. H. Benbouzid, "Current frequency spectral subtraction and its contribution to induction machines' bearings condition monitoring," IEEE Trans. on Energy Conversion, vol. 28, no. 1, pp. 135-144, March 2013.

[18] A. Ceban, R. Pusca, and R. Romary, "Study of rotor faults in induction motors using external magnetic field analysis," IEEE Transactions on Industrial Electronics, vol. 59, no. 5, pp. 2082-2096, May 2012.

[19] H. Toliyat and T. Lipo, "Transient analysis of cage induction machines under stator, rotor bar and end ring faults," IEEE Transactions on Energy Conversion, vol. 10, no. 2, pp. 241-247, 1995.

[20] P. Zhang, Y. Du, T. Habetler, and B. Lu, "A survey of condition monitoring and protection methods for medium-voltage induction motors," IEEE Transactions on Industry Applications, vol. 47, no. 1, pp. 34-46, January/February 2011.

[21] A. H. Bonnett and C. Yung, "Increased efficiency versus increased reliability," IEEE Industry Applications Magazine, vol. 14, no. 1, January/February 2008. 